\title{
THE EFFECT OF POSTURE ON THE LUMBAR SPINE
}

\author{
M. A. ADAMS, W. C. HUTTON
}

From the Polytechnic of Central London

\begin{abstract}
A series of experiments showing how posture affects the lumbar spine is reviewed. Postures which flatten (that is, flex) the lumbar spine are compared with those that preserve the lumbar lordosis. Our review shows that flexed postures have several advantages: flexion improves the transport of metabolites in the intervertebral discs, reduces the stresses on the apophyseal joints and on the posterior half of the annulus fibrosus, and gives the spine a high compressive strength. Flexion also has disadvantages: it increases the stress on the anterior annulus and increases the hydrostatic pressure in the nucleus pulposus at low load levels.

The disadvantages are not of much significance and we conclude that it is mechanically and nutritionally advantageous to flatten the lumbar spine when sitting and when lifting heavy weights.
\end{abstract}

Current ideas on what constitutes "good posture" are rather vague. The usual advice, possibly based on aesthetic and military traditions, is "sit up straight" and "don"t slouch". Paradoxically, sitting up straight is taken to mean sitting with a lumbar lordosis and not allowing the lumbar spine to flex and flatten its curve.

As far as the lumbar spine is concerned, there is no reliable evidence that sitting up straight is, in fact, beneficial. On the contrary, population studies have shown that lumbar disc degeneration is rare among people who habitually sit or squat in postures which flatten the lumbar spine (Fahrni and Trueman 1965). Such postures are instinctively assumed by children and by many adults. If these natural preferences are to be discouraged and advice given on posture, then such advice should be founded on scientific evidence. To this end we have carried out a series of experiments on cadaveric lumbar spines to show how posture can affect both the mechanics of the lumbar spine and also the nutritional status of the intervertebral discs.

\section{METHOD}

The normal curves of the spine help it to act as a shock absorber by reducing its longitudinal stiffness (by a similar mechanism a metal rod can absorb shock if it is curved to form a helical spring). These spinal curves are absent in the child but pronounced in the adult in all human populations: they therefore must be considered "natural".

The lumbar curvature is defined in Figure 1. In the

M. A. Adams. PhD. Research Fellow

W. C. Hutton. MSc. Professor of Biomechanics, School of Engineering and Science

Polytechnic of Central London. 115 New Cavendish Street, London WIM 8JS. Fngland.

Requests for reprints should be sent to Professor Hutton.

( 1985 British Editorial Society of Bone and Joint Surgery

$0301620 \times 854130 \$ 2.00$

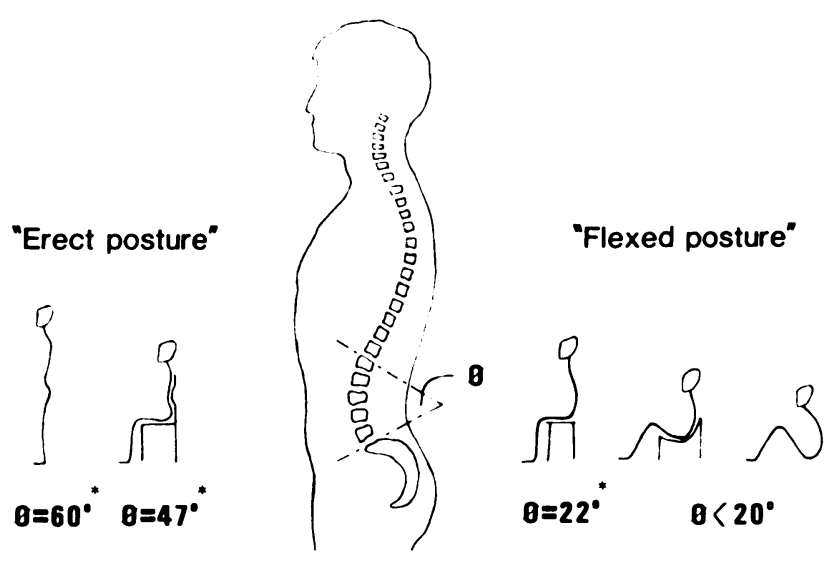

Fig. 1

The lumbar curvature $(\theta)$ is defined as the angle between the upper surfaces of the sacrum and the LI vertebral body. Values of $\theta$ are shown for typical erect and flexed postures $\left({ }^{*}\right.$ calculated from Andersson $e t$ al. 1979).

unstressed spine it averages about 40 , as measured from the dissected spines of cadavers (Farfan, Huberdeau and Dubow 1972). In a living adult, the curvature varies with posture, from 80 when leaning backwards to 0 when bending fully forwards (calculated from Pearcy, Portek and Shepherd 1984). Typical values within this range for standing and sitting postures are shown in Figure 1. Three of these values are calculated from Andersson et al. (1979), and the fourth is from our own measurements.

We have defined postures according to their effect on the lumbar curvature: "flexed postures" are those that flatten the lumbar spine and produce low values of lumbar curvature, whereas "erect postures" are those that maintain or increase the lumbar curvature.

Posture and the loading of the apophyseal joints. These joints stabilise the spine and protect the discs from both excessive flexion (Adams, Hutton and Stott 1980) and axial rotation (Adams and Hutton 1981). They also play a major role in resisting shear and compressive forces, although this varies considerably with posture. 


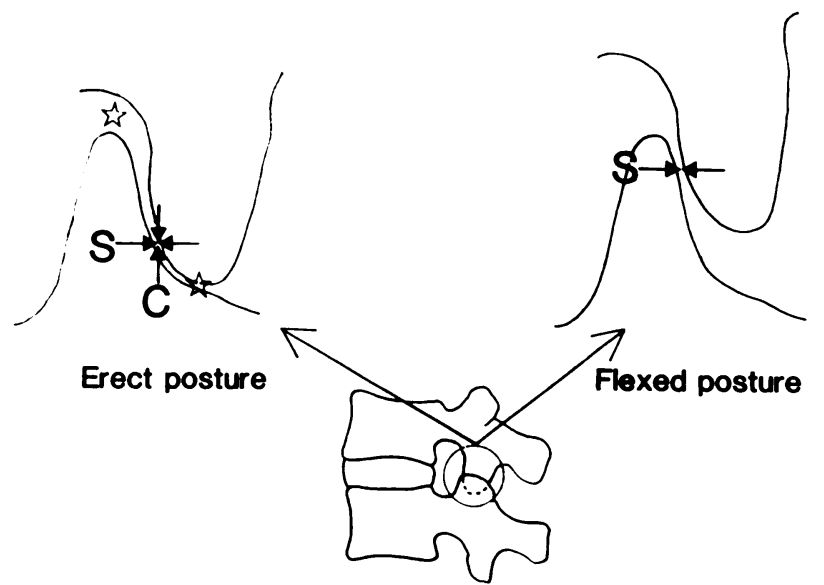

Fig. 2

The effect of posture on the loading of the apophyseal joints. In the erect posture the articular surfaces resist the intervertebral shear force (S) and a proportion of the intervertebral compressive force $(C)$. Extraarticular impingement can also occur (as shown by the star). In the flexed posture the articular surfaces resist the shear force but play no part in resisting the compressive force.

In the erect posture, the apophyseal joints resist most of the shear force acting on the spine (Hutton, Stott and Cyron 1977) as well as about $16 \%$ of the compressive force (Adams and Hutton 1980). The resulting stress between the articular surfaces is concentrated in the lower margins of the joint (Dunlop, Adams and Hutton 1984) as shown in Figure 2. If the disc is unusually narrow and degenerate, then the facets may come into close apposition and may then resist up to $70 \%$ of the compressive force on the spine (Adams and Hutton 1980). Much of this abnormally high resistance is due to extraarticular impingement of the facet tips on the adjacent lamina or pedicle (Dunlop, Adams and Hutton 1984). In such cases, the apophyseal joints show gross osteoarthritic changes (Adams and Hutton 1980).

In the flexed posture, the apophyseal joints resist the shear force but now play no part in resisting the intervertebral compressive force (Adams and Hutton 1980). The stress between the articular surfaces is lower than in the erect posture and is concentrated in the midd!e and upper parts of the joint (Dunlop, Adams and Hutton 1984) as shown in Figure 2. In the flexed posture there is no extra-articular impingement.

Posture and the loading of the intervertebral disc. The intervertebral discs and vertebral bodies comprise the main weight-bearing column of the lumbar spine. Posture affects the way this column resists the loads applied to it, but has little effect on the magnitude of these loads (see below: posture and muscle activity).

Under load, an unwedged disc tends to behave as a hydrostatic body exerting a uniform compressive stress on the vertebral end-plates (Horst and Brinckmann 1981). By wedging a disc we complicate this slightly: young non-degenerate discs remain hydrostatic, but mature and degenerate discs sustain pressure gradients. This means that when a mature disc is wedged in the erect posture, the highest compressive stresses are transmitted through the posterior annulus and the lowest through the anterior annulus (Horst and Brinckmann 1981). Similarly, in flexed postures the highest compressive stresses are transmitted through the anterior annulus and the lowest through the posterior annulus.

In all but the most degenerate discs, the nucleus pulposus exhibits hydrostatic pressure, the magnitude of which is dependent on disc wedging (Nachemson 1963). At low load levels (appropriate in relaxed sitting and standing postures), pressure in the nucleus pulposus for the same applied compressive load is about $50 \%$ higher in the "flexed" position than in the "erect" position (calculated from Nachemson, Schultz and Berkson 1979). An explanation of this is given in the appendix.

The effects of posture on the mechanics of the mature intervertebral disc are illustrated in Figures 3 and 4.

Posture and the nutrient supply to the intervertebral discs. The lumbar discs are the largest avascular structures in the body. Nutrients reach their cells by two routes: from the blood vessels in the vertebral bodies and from tissue fluid surrounding the annulus fibrosus. There are two transport processes for each route-fluid flow and diffusion-and both of these are affected by posture.

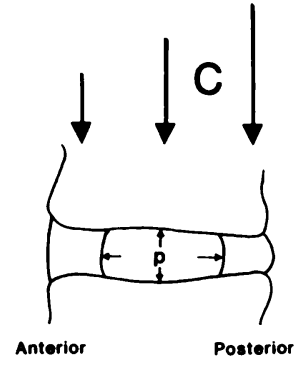

Erect posture

Fig. 3

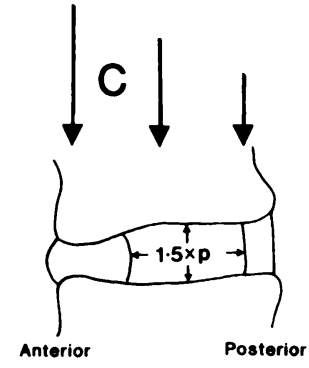

Flexed posture

Fig. 4
The effect of posture on the mature intervertebral disc. In the erect postures (Fig. 3) the compressive force (C) produces the highest stress on the posterior annulus, while in the flexed posture (Fig. 4) the stress is highest on the anterior annulus and the hydrostatic pressure $(p)$ in the nucleus is increased.

Fluid flow is caused by pressure changes on the disc. High pressure causes fluid to be expelled from the disc, while low pressure (lying down, for example) allows the proteoglycans in the disc to suck in fluid from surrounding tissue. Flexed postures increase this fluid exchange because they cause more fluid to be expelled from the disc than do erect postures (Adams and Hutton 1983). The effect is particularly marked in the nucleus pulposus (Fig. 5).

Diffusion occurs as a result of a chemical concentration gradient: nutrients diffuse into the disc, and waste products diffuse out of it. In erect postures diffusion occurs more readily into the anterior annulus than into the posterior annulus (Fig. 5), probably because the disc height decreases from front to back (Adams and Hutton 


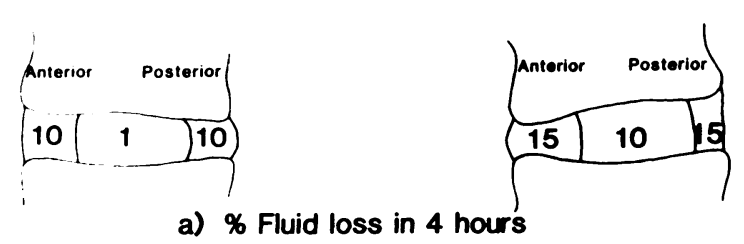

Erect posture

Flexed posture

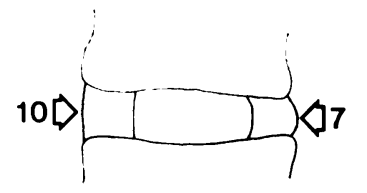

b) Diffusion of solute into disc

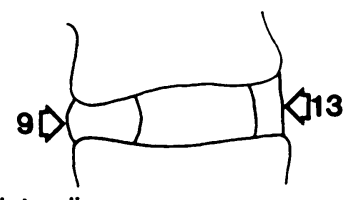

Fig. 5

The percentage of fluid loss (a) from the disc depends on the posture. The three values for each disc refer to the anterior annulus, the nucleus pulposus and the posterior annulus. In the erect posture solutes can diffuse more easily into the anterior half of the disc than into the posterior half: this imbalance is reversed in the flexed posture. The numbers are proportional to the amount of solute (b) that can diffuse during four hours.

1984). Flexed postures reverse this and the overall rate of diffusion into the disc is increased. These results can be explained by the way flexed posture deforms the annulus (Adams and Hutton 1984).

Posture and muscle activity. Trunk muscle activity is required to stabilise the spine and prevent it from buckling (Morris, Lucas and Bresler 1961); obviously, the need for stability is greatest when the spine is vertical and least when it is horizontal. This explains why the activity of the back muscles is reduced when the backrest of a chair is made to incline more (Andersson et al. 1975). However, for a given backrest angle, posture (as defined by lumbar curvature) has only a minor effect on muscle activity (Andersson et al. 1975). Figure 6 illustrates how trunk muscle activity is determined by the need to stabilise the spine rather than by the lumbar curvature.

Posture and spinal injury. In the previous sections we have considered typical standing and sitting postures with fairly low static loads on the spine. In this section we
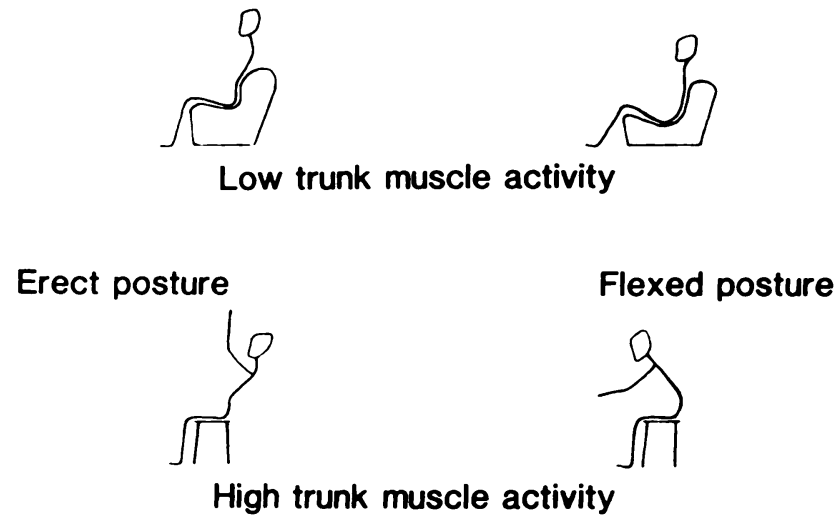

Fig. 6

Trunk muscle activity is determined by the need to stabilise the spine rather than by the lumbar curvature. In the upper figures the spine is supported and there is low trunk muscle activity, whereas in the lower figures the spine is unsupported and there is high trunk muscle activity. consider high dynamic loading of the spine and how such loads can produce different injuries in different postures.

Lifting heavy weights requires high back muscle activity and results in a high compressive force on the spine. For young men lifting with maximum effort, this force can reach 8000-9000 N (Hutton and Adams 1982). The effect of such forces on the lumbar spine has been tested on cadaveric lumbar motion segments (two adjacent vertebrae and the intervening disc and ligaments); the outcome depends very much on posture. If the motion segment is compressed without being flexed, there may be damage to the vertebral body or end-plate (Perey 1957). If it is wedged in a flexed posture (Fig. 7), it should not be damaged at all (Hutton and Adams 1982). However, if flexion is excessive (hyperflexion) so that the posterior intervertebral ligaments are overstretched, then the motion segment is again vulnerable; the anterior vertebral body may be crushed or there may be sudden posterior prolapse of the intervertebral disc (Adams and Hutton 1982).
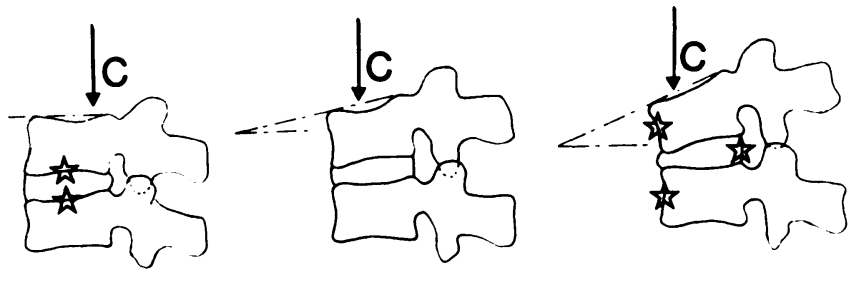

Erect posture

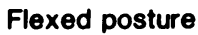

Hyperflexion

Fig. 7

The effect of posture on spinal injury. In the erect posture, the compressive force on the spine $(C)$ can cause end-plate fracture. In the flexed posture the spine has a high compressive strength. In hyperflexion $C$ can cause posterior disc prolapse or damage to the anterior vertebral body.

Activities such as digging or repeated heavy lifting subject the lumbar spine to fatigue compressive loading. The effect of such loading has been investigated by testing cadaveric motion segments to destruction and, again, the results depend on posture. If the motion segment is not flexed, the site of failure is in the vertebral body and end-plate (Hardy et al. 1958). If it is flexed, the lamellae of the annulus can become distorted and this sometimes leads to radial fissures in the disc (Adams and Hutton 1983).

\section{DISCUSSION}

The advantages and disadvantages of flexing the lumbar spine are summarised in Table I. Let us first consider the advantages.

Reducing the high stresses that can be found on the tips of the facet joints may well be significant. In a lordotic posture, the stress between the facet surfaces can exceed the peak levels found in the articular cartilage of the hip and knee (Dunlop, Adams and Hutton 1984) and may be responsible for the very high incidence of osteoarthritis in these joints (Lewin 1964; Adams and 
Table I. Flexed posture

Adramlages

Reduced stresses at the apophyseal joints

Reduced compressive stress on the posterior annulus

Improved transport of disc metabolites

High compressive strength of the spine

Disadramtage's

Increased compressive stress on the anterior annulus

Increased hydrostatic pressure in the nucleus at low load levels

Hutton 1980). Also, the possible extra-articular impingement between the facet tip and the adjacent lamina or pedicle in a lordotic posture could be a source of low back pain, especially if the joint capsule is trapped between the bony surfaces.

Similarly, reducing the compressive stress on the posterior annulus may be of clinical significance, since this stress causes outward bulging of the posterior annulus into the spinal canal and intervertebral foramina (Brown, Hansen and Yorra 1957). In many people, this may lead to compression of the dural sac and cause pain (Penning and Wilmink 1981). There is also evidence from animal experiments that high compressive loading of the posterior annulus can eventually lead to degenerative changes in this part of the disc (Lindblom 1957).

Improving the metabolite transport in the disc must be of real value since its overall supply of glucose has been described as barely adequate (Maroudas et al. 1975). Moreover, the part of the disc with the most critical supply, the inner posterior annulus (Nachemson 1976), is precisely the part most helped by a flexed posture (Adams and Hutton 1984). Deficient metabolite transport has been linked with degenerative changes in the disc (Nachemson et al. 1970; Holm and Nachemson 1982).

Although the high compressive strength of a flexed lumbar spine is probably important, the compressive force in this area can nonetheless be reduced in life by intra-abdominal pressure (Bartelink 1957). However, flattening (flexing) the lumbar spine gives it an extra margin of safety, which may be important in activities involving repeated bending and lifting. The increased compressive strength of a flexed lumbar spine may be explained by the non-linear elastic behaviour of the disc. as outlined in the appendix.

Neither of the disadvantages listed in Table I seems to be of much significance. The anterior annulus is the thickest and stiffest part of the annulus (Galante 1967) and is evidently well capable of resisting the stresses applied to it since it is usually the last part of the disc to exhibit degenerative changes (Ritchie and Fahrni 1970).

As for the second disadvantage (increased hydrostatic pressure in the nucleus pulposus), this may seem more serious until it is realised that the pressure increase has been demonstrated only at low load levels, where there is no likelihood of mechanical damage: in sitting or standing postures, the compressive force on the lumbar spine is about $1000 \mathrm{~N}$. or less than one-tenth of the force required to cause failure (Hutton and Adams 1982).

There is one other possible objection to the flexed posture: because flexion can lead to disc damage under fatigue loads (see posture and spinal injury section), it might lead to similar damage when the load levels are low but persist for long periods of time. Generally, a structure can suffer fatigue damage at low load levels provided enough loading cycles are applied. However, there is always a "cut-off" value of load, below which no damage accumulates no matter how long that load is applied. In the case of the lumbar discs, the "cut-off" load is probably above that normally encountered in flexed sitting postures, since people who habitually sit with their lumbar spines flexed have less disc disease (Fahrni and Trueman 1965); it thus seems unlikely that the flexed sitting posture could be damaging to their discs.

In this paper we have investigated the effects of posture on a normal, symptom-free spine: our conclusions do not necessarily apply to the spine of a person with back pain. On balance, however, it is mechanically and nutritionally advantageous to flatten the lumbar spine when sitting and when lifting heavy weights.

\section{APPENDIX}

"Non-linear" elastic behaviour of the disc. In order to explain why wedging a disc in flexion increases the pressure in the nucleus pulposus, and why a flexed spine has a high compressive strength, a simple model of the disc is presented.

Figure 8 shows a disc as three parallel springs representing respectively the anterior annulus, the nucleus pulposus and the posterior annulus. These springs are "non-linear", that is, they become stiffer the more they are compressed or stretched. In the figure, the width of each spring is drawn in proportion to the force acting on it.

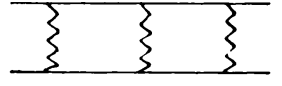

a)

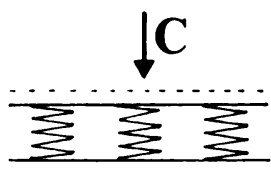

b) Erect posture
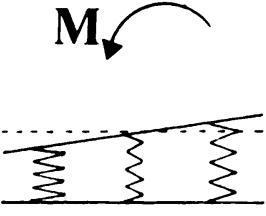

c)

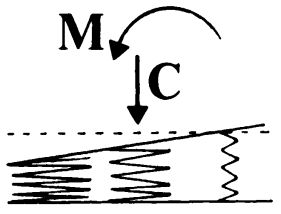

d) Flexed posture
Fig. 8

"Non-linear" elastic behaviour of the disc, illustrating the effects of bending $(\mathrm{M})$ and of compressive force $(\mathrm{C})$. 
Figure 8a shows the disc under no external forces nor with any bending moment acting on it. In Figure $8 b$ the disc is subjected to a compressive force $(C)$ but with no bending moment (as in the erect posture). In Figure $8 \mathrm{c}$ the disc is subjected to a bending moment $(\mathrm{M})$ but no compressive force; here the centre of rotation lies in the posterior half of the disc (Rolander 1966) so that the bending moment is resisted by the anterior annulus and the nucleus in compression and by the posterior annulus in tension. In Figure $8 \mathrm{~d}$, the bending moment and compressive force are combined, as in a flexed posture. Comparing $8 \mathrm{~d}$ with $8 \mathrm{~b}$ we see that pressure in the nucleus pulposus is higher in flexed postures.

The above only applies to low values of $\mathrm{C}$, appli- cable to standing and sitting postures. At high values of $\mathrm{C}$, applicable to lifting, the non-linearity of the springs becomes important and we can now explain the high compressive strength of a flexed spine (see Fig. 8d). The anterior annulus "spring" is considerably shortened and, because of non-linearity, will be stiffer than the nuclear spring. If compressive force $(\mathrm{C})$ is increased to high levels, the additional load will be borne primarily by the anterior annulus, to the advantage of the nucleus. By this mechanism the pressure in the nucleus is prevented from rising to levels that would fracture the weak central portion of the end-plate. When high loads are applied in the erect posture (b), this mechanism does not occur and the spine is manifestly weaker.

\section{REFERENCES}

Adams MA, Hutton WC. The effect of posture on the role of the apophysial joints in resisting intervertebral compressive forces. J Bome Joint Surg [Br] 1980:62 B:358 62.

Adams MA, Hutton WC. The relevance of torsion to the mechanical derangement of the lumbar spine. Spine 1981:6:241-8.

Adams MA, Hutton WC. Prolapsed intervertebral disc: a hyperflexion injury. Spine 1982:7:184 91.

Adams MA, Hutton WC. The effect of posture on the fluid content of lumbar intervertebral discs. Spine 1983:8:665 71.

Adams MA. Hutton WC. The effect of fatigue on the lumbar intervertebral disc. J Bone Joint Surg [Br] 1983;65-B: 199-203.

Adams MA, Hutton WC. The effect of posture on diffusion into lumbar intervertebral discs. Orthop Trans 1984:8:415 6.

Adams MA, Hutton WC, Stott JRR. The resistance to flexion of the lumbar intervertebral joint. Spine 1980:5:245-53.

Andersson BJ, Örtengren R, Nachemson AL, Elfstrom G, Broman H. The sitting posture: an electromyographic and discometric study. Orthop Clin North Am 1975:6(1): 105-20.

Andersson GBJ, Murphy RW, Ortengren R, Nachemson AL. The influence of backrest inclination and lumbar support on lumbar lordosis. Spine 1979:4:52-8.

Bartelink DL. The role of abdominal pressure in relieving the pressure on the lumbar intervertebral discs. J Bone Joint Surg $[B r]$ 1957:39 B: 71825 .

Brown T, Hansen RJ, Yorra AJ. Some mechanical tests on the lumbosacral spine with particular reference to the intervertebral discs: a preliminary report. J Bome Joint Surg $[\mathrm{Am}]$ 1957:39-A:1135 64 .

Dunlop RB, Adams MA, Hutton WC. Disc space narrowing and the lumbar facet joints. J Bone Joint Surg [Br] 1984;66-B:706-10.

Fahrni WH, Trueman GE. Comparative radiological study of the spines of a primitive population with North Americans and Northern Europeans. J Bone Joint Surg [Br] 1965:47 - B:552-5.

Farfan HF, Huberdeau RM, Dubow HI. Lumbar intervertebral disc degeneration. J Bone Joint Surg $[\mathrm{Am}]$ 1972:54-A:492-510.

Galante JO. Tensile properties of the human lumbar annulus fibrosus. Acta Orthop Scand 1967:Suppl 100.

Hardy WG, Lissner HR, Webster JE, Gurdjian ES. Repeated loading tests of the lumbar spine: a preliminary report. Surg Forum 1958; 9:690 5 .
Holm S, Nachemson A. Nutritional changes in the canine intervertebral disc after spinal fusion. Clin Orthop 1982:169:243 58.

Horst S, Brinckmann P. Measurement of the distribution of axial stress on the end-plate of the vertebral body. Spine 1981;6:217 32 .

Hutton WC, Adams MA. Can the lumbar spine be crushed in heavy lifting? Spine 1982:7:586 90.

Hutton WC, Stott JRR, Cyron BM. Is spondylolysis a fatigue fracture? Spine 1977:2:2029.

Lewin T. Osteoarthritis in lumbar synovial joints: a morphological study. Acta Orthop Scand 1964: Suppl 73.

Lindblom K. Intervertebral-disc degeneration considered as a pressure atrophy. J Bone Joint Surg [Am] 1957:39 A:933 45.

Maroudas A, Stockwell RA, Nachemson A, Urban J. Factors involved in the nutrition of the human lumbar intervertebral disc: cellularity and diffusion of glucose in vitro. J. Anat 1975:120:113-30.

Morris JM, Lucas DB, Bresler B. Role of the trunk in stability of the spine. J Bone Joint Surg $[\mathrm{Am}]$ 1961:43 A:327 51 .

Nachemson AL. The influence of spinal movements on the lumbar intradiscal pressure and on the tensile stresses in the annulus fibrosus. Acta Orthop Scand 1963:33:183 207.

Nachemson A, Lewin T, Maroudas A, Freeman MAR. In vitro diffusion of dye through the end-plates and the annulus fibrosus of human inter-vertebral discs. Acta Orthop Scand 1970:41:589-607.

Nachemson AL. The lumbar spine: an orthopaedic challenge. Spine 1976:1:59-71.

Nachemson AL, Schultz AB, Berkson MH. Mechanical properties of human lumbar spine motion segments: influences of age, sex, disc level and degeneration. Spine 1979:4:1 8.

Pearcy M, Portek I, Shepherd J. Three-dimensional X-ray analysis of normal movement in the lumbar spine. Spine 1984:9:294 7.

Penning L, Wilmink JT. Biomechanics of lumbosacral dural sac: a study of flexion-extension myelography. Spine 1981:6:398-408.

Perey 0 . Fracture of the vertebral endplate in the lumbar spine: an experimental biomechanical investigation. Acta Orthop Scand 1957: Suppl 25.

Ritchie JH, Fahrni WH. Experimental surgery: age changes in lumbar intervertebral discs. Can J Surg 1970:13:65 71.

Rolander SD. Motion of the lumbar spine with special reference to the stabilizing effect of posterior fusion: an experimental study on autopsy specimens. Acta Orthop Scand 1966: Suppl 90. 